\title{
Epistasis between neurochemical gene polymorphisms and risk for ADHD
}

\author{
Ricardo Segurado $^{\star, 1}$, Mark A Bellgrove ${ }^{2}$, Francesca Manconi ${ }^{3}$, Michael Gill ${ }^{1}$ and Ziarah Hawi ${ }^{1,2}$
}

A number of genes with function related to synaptic neurochemistry have been genetically associated with attention deficit/ hyperactivity disorder. However, susceptibility to the development of common psychiatric disorders by single variants acting alone, can so far only explain a small proportion of the heritability of the phenotype. It has been postulated that the unexplained 'dark heritability' may at least in part be due to epistatic effects, which may account for the small observed marginal associations, and the difficulties with replication of positive findings. We undertook a comprehensive exploration of pair-wise interactions between genetic variants in 24 candidate genic regions involved in monoaminergic catabolism, anabolism, release, re-uptake and signal transmission in a sample of 177 parent-affected child trios using a case-only design and a case-pseudocontrol design using conditional logistic regression. Marker-pairs thresholded on interaction odds ratio (OR) and $P$-value are presented. We detected a number of interaction $0 R s>4.0$, including an interesting correlation between markers in the $A D R A 1 B$ and $D B H$ genes in affected individuals, and several further interesting but smaller effects. These effects are no larger than you would expect by chance under the assumption of independence of all pair-wise relations; however, independence is unlikely. Furthermore, the size of these effects is of interest and attempts to replicate these results in other samples are anticipated.

European Journal of Human Genetics (2011) 19, 577-582; doi:10.1038/ejhg.2010.250; published online 2 February 2011

Keywords: ADHD; epistasis; candidate gene

\section{INTRODUCTION}

The heritability of attention deficit/hyperactivity disorder (ADHD) is well established, and genetic association data have been reviewed and meta-analysed recently by Gizer et al. ${ }^{1}$ The detection of DNA variants, which increase risk for this disorder is important for biochemical and pharmacological research into this disorder, and may permit the facilitation of diagnosis, or refinement of the phenotype on the basis of a biological marker. Specifically, association between monoaminergic genes and ADHD is an active area of investigation, stimulated principally by the mode of action of current pharmacological treatments for the disorder, primary among which is methylphenidate, a known dopamine (DA) and noradrenaline (NA) re-uptake inhibitor. Associations between variants at the DA, serotonin and NA transporter and receptor genes, with ADHD, have been investigated, and meta-analyses support the presence of ADHD risk alleles with small to moderate effects at the DA receptor $(D R D) 4$, $D R D 5$ and SLC6A3 genes ${ }^{1-3}$ in candidate gene studies, and modest evidence at $A D R A 1 A, T P H 2$ and $D D C$ from a recent meta-analysis of genome-wide association studies. ${ }^{4}$

The influence of higher order interactions between genes has been posited for common, complex genetic disorders on the basis of biological plausibility, and the presence of interactions influencing gene expression and protein function, both in model organisms and in human disease susceptibility. ${ }^{5,6}$ In the case of ADHD, the most likely known risk genes inhabit the same biochemical neighbourhood (see for example refs. 7,8 ) in that the functions of each gene product are related directly via protein-protein interaction or indirectly via regulatory pathways. Therefore, there are grounds for prior supposition that common genetic variants in several risk genes may act synergistically to influence disease risk. Methods for detecting interaction, or epistasis, in case-control samples have received much attention recently, ${ }^{9,10}$ particularly in the rapidly developing areas of machine learning. Methods for family-based samples are less well developed - usually adaptations of case-control methods (eg, using matched case-pseudocontrol samples). However, in addition to large family-based samples being used in the latest generation of genemapping studies (eg, the Autism Genome Project; ${ }^{11}$ IMAGE $^{12}$ ), family-based samples were extensively used in candidate gene experiments before the emergence of genome-wide association studies. These are excellent genotype resources for exploring epistasis, as these studies have genotyped a relatively small number of genetic polymorphisms within strong candidate genes.

The aim of this study was to use a subset of genetic markers which we expect to be enriched for true involvement in pathogenesis of ADHD, to (1) attempt to detect any large epistatic effects which may exist and (2) explore epistatic effect sizes between candidate genes, for prioritisation of further investigation in this sample and replication in other samples. To this end, a limited number of variants in a range of strong candidate genes for ADHD were selected (see Methods). As this study was a focused exploration of interaction, we elected to use two principal techniques to analyse the data thoroughly for various forms of interaction effects. Thus, first, a case-only design was adopted

\footnotetext{
${ }^{1}$ Neuropsychiatric Genetics Group, Department of Psychiatry, Trinity College Dublin, Dublin, Ireland; ${ }^{2}$ Queensland Brain Institute and School of Psychology, The University of Queensland, Brisbane, Queensland, Australia; ${ }^{3}$ Department of Neurosciences ‘B B Brodie', University of Cagliari, Cagliari, Italy

*Correspondence: Dr R Segurado, Neuropsychiatric Genetics Group, Department of Psychiatry, Institute for Molecular Medicine, Trinity Centre for Health Sciences, St James' Hospital, Dublin 8, Ireland. Tel: +353 18963405; Fax: +353 18968461; E-mail: ricardo.segurado@tcd.ie

Received 13 April 2010; revised 30 November 2010; accepted 9 December 2010; published online 2 February 2011
} 
to detect association between unlinked genes in affected individuals. This allows calculation of an 'interaction odds ratio (OR)' equivalent to the interaction relative risk under the assumption of independence of the genes in the general population. ${ }^{13}$ Second, a similar model was fitted to binary affection status by logistic regression. ${ }^{14}$ This method examines patterns of transmission of alleles from each of the markers in a pair, thus is robust to bias arising from population stratification.

\section{METHODS}

\section{Recruitment and sample description}

A total of 177 children and their families participated in the study including 5 families with 2 affected children. The families were recruited from child guidance clinics, ADHD support groups and via direct clinician referral. A stringent diagnosis of DSM-IV and/or ICD10 ADHD was made as described by Kirley et al, ${ }^{15}$ using the Child and Adolescent Psychiatric Assessment (CAPA) and the Teacher Telephone Interview (TTI). Exclusion criteria included epilepsy, fragile X syndrome, fetal alcohol syndrome, pervasive developmental disorder, Tourette's syndrome, psychosis or IQ $<70$. Affected children met research criteria for ICD-10 hyperkinetic disorder (100 children) and/or DSMIV ADHD (combined: 112, inattentive: 21 or hyperactive-impulsive: 11 subtypes), and/or a clinical diagnosis of ADHD not meeting full research criteria but deemed to lie on the broad ADHD phenotypic spectrum. Therefore, the present sample consisted of 172 parent - affected child trios.

\section{Marker selection and genotyping}

Gene selection began with a set of candidate genes previously typed in this sample, and was expanded to include several genes found to be associated in other samples and genes within neurochemical systems plausibly linked to ADHD (Table 1; Supplementary Tables S1 and S2). This study was limited to 25 autosomal genes (CES1 and SLC6A2 were treated as one unit because of physical proximity). Markers within these genes were either (i) previously

Table 1 Description of candidate genes typed and marker coverage. Spacing based on human genome build hg18

\begin{tabular}{|c|c|c|c|c|c|}
\hline \multirow[b]{2}{*}{ Gene } & \multirow[b]{2}{*}{ Chr } & \multicolumn{2}{|c|}{ Position (bp) } & \multirow{2}{*}{$\begin{array}{l}\text { No. of } \\
\text { markers }\end{array}$} & \multirow{2}{*}{$\begin{array}{l}\text { Mean marker } \\
\text { spacing (bp) }\end{array}$} \\
\hline & & Start & Stop & & \\
\hline$A D R A 2 B$ & 2 & 96142350 & 96145615 & 1 & - \\
\hline$A D R A 2 C$ & 4 & 3738094 & 3740051 & 1 & - \\
\hline DRD5 & 4 & 9392356 & 9394731 & 3 & 663473 \\
\hline$A D R A 1 B$ & 5 & 159276318 & 159332595 & 11 & 4433 \\
\hline ADRB2 & 5 & 148186349 & 148188381 & 2 & 33 \\
\hline$S \angle C 6 A 3$ & 5 & 1445910 & 1498538 & 2 & 17933 \\
\hline$H T R 1 B$ & 6 & 78228667 & 78229839 & 5 & 470 \\
\hline$D D C$ & 7 & 50493628 & 50600648 & 2 & 3744601 \\
\hline ADRA1A & 8 & 26661584 & 26778839 & 14 & 8550 \\
\hline$D B H$ & 9 & 135491306 & 135514287 & 11 & 2315 \\
\hline$A D R A 2 A$ & 10 & 112826780 & 112830652 & 3 & 1859 \\
\hline SLC18A2 & 10 & 118990706 & 119027085 & 5 & 9173 \\
\hline DRD4 & 11 & 627305 & 630703 & 5 & 1011 \\
\hline$T H$ & 11 & 2141735 & 2149611 & 1 & - \\
\hline TPH1 & 11 & 17998660 & 18018911 & 3 & 3526 \\
\hline TPH2 & 12 & 70618893 & 70712488 & 12 & 6266 \\
\hline HTR2A & 13 & 46305514 & 46369170 & 8 & 8701 \\
\hline$S L C 6 A 2$ & 16 & 54248057 & 54295201 & 9 & 6526 \\
\hline CES1 & 16 & 54394265 & 54424576 & 3 & 22743 \\
\hline PNMT & 17 & 35078033 & 35080254 & 3 & 3093 \\
\hline SLC6A4 & 17 & 25547506 & 25587080 & 5 & 9879 \\
\hline ADRA1D & 20 & 4149278 & 4177659 & 11 & 2359 \\
\hline SNAP25 & 20 & 10147477 & 10236065 & 16 & 6019 \\
\hline COMT & 22 & 18309263 & 18337496 & 5 & 6396 \\
\hline
\end{tabular}

characterised in this sample; (ii) previously associated in other samples; (iii) selected because of known or suspected functional relevance (on gene or protein expression, protein sequence, and including presence in a known promoter region and lying within 500 base pairs of an exonic boundary); or (iv) to tag the remainder of the gene $\left(r^{2} \geq 0.8\right)$ insofar as gene size and marker density made possible. Markers and genes were placed on the human genome 18 physical map (hg18; NCBI build 36.3) using dbSNP (http://www.ncbi.nlm. nih.gov/SNP/) or by linear interpolation from flanking markers.

A total of 179 markers were genotyped. Single nucleotide polymorphisms (SNPs) were genotyped commercially at K-Bioscience using a competitive allele-specific PCR - KASPar (K-Bioscience, Hoddesdon, UK; personal communication), or Taqman chemistries (Applied Biosystems, Foster City, CA, USA). Three markers, at the DBH (rs1611115 and rs6271) and catechol-Omethyltransferase (COMT; rs4680) genes were genotyped in-house with Taqman assays on a 7900HT sequence analyzer (Applied Biosystems). Microsatellite and minisatellite markers were typed in-house as described previously; these included the markers in SLC6A3 and DRD $5,{ }^{16} D R D 4,{ }^{17} T H^{18}$ and SLC6A4. ${ }^{19}$

Multiallelic ( $>2$ alleles) markers were collapsed into two categories on the basis of (1) bimodality of the allele frequency histogram in founder individuals and (2) so as to approximate equifrequency of the down-coded 'alleles'. This might be expected to reflect the genetic history of the microsatellite. We did not use previously associated alleles at these markers as a basis for recoding. ${ }^{20}$

Only autosomal genetic markers with a minor allele frequency in founders of $>0.05$ were retained; only markers with over $80 \%$ genotype rate were included; Pedcheck ${ }^{21}$ was used to detect Mendelisation errors, genotypes showing errors were removed for the entire trio; finally, markers which showed extreme deviation from Hardy-Weinberg equilibrium in the parents were excluded because of the possibility of miscalled genotypes. When markers within the same gene were in near perfect linkage disequilibrium in founders $\left(r^{2} \geq 0.98\right)$, the one with least complete genotyping was discarded.

\section{Data analysis}

An allelic case-only test for pair-wise interaction of $\mathrm{SNPs}^{22}$ was performed using the proband from each pedigree. To exclude the effect of linkage disequilibrium between SNPs, all intragenic SNP pairs and all intergenic SNP pairs separated by $<500 \mathrm{~kb}$ were excluded. Tables with any zero cell count were discarded. A $\chi^{2}$-test ( 1 d.f.) was performed on the $2 \times 2$ tables, and an OR estimate was calculated.

The null distribution of the OR for each pair of SNPs examined was simulated by fixing genotypes at the first marker and permuting genotypes at the second. Two-sided $P$-values for the ORs were estimated from 50000 permutations or from 1000000 permutations for marker pairs which showed an initial empirical $P$-value $<0.001$. The $95 \%$ confidence intervals for the interaction ORs were generated from 1000 bootstrap samples for each marker pair. A global, family-wise $P$-value was also calculated from the proportion of the 50000 permutations, in which an OR was exceeded in any of the pair-wise tests.

Interactions were also tested in a logistic regression model, implemented on a matched case-pseudocontrol sample. ${ }^{14}$ A total of 15 pseudocontrols were generated for each trio, for each marker pair, from all combinations of parental two-marker genotypes. Two models were fitted to the data in R (http://cran. r-project.org) with the coxph function from the survival package, using robust variance estimation, stratified on a family ID variable (equivalent to a conditional logistic regression). An interaction parameter was tested by contrasting the model with a nested model containing main effects alone using a likelihood ratio $\chi^{2}$-test with one degree of freedom. Only additive allelic effects were examined. For each marker pair, perfectly collinear variables were handled by removing one from the model. A pseudo- $\mathrm{R}^{2}$ for each model representing how much of the variance in the dependent variable (affection status) is explained by the genetic terms was also calculated.

\section{RESULTS}

Twenty-seven markers were excluded (see Methods) owing to low genotyping rate, 4 to low minor allele frequency in parents and 3 to extreme deviation from Hardy-Weinberg equilibrium in parents (goodness-of-fit $\chi^{2}, P<0.00033=0.05 / 177$ ). Interaction between one 
pair of genes (CES1-SLC6A2) was not examined because of physical proximity $<500 \mathrm{kbp}$. Four markers were removed owing to near perfect linkage disequilibrium in parents with neighbouring markers $\left(r^{2}=0.98-1.00\right)$. Table 1 shows genic positions, marker number and spacing. The $\chi^{2}$-test statistics for syntenic markers had very low correlation with the intermarker distance (Supplementary Figure S3).

The remaining 141 markers had a mean missing genotype rate of 0.075 in affected individuals. We estimated from single-marker missing genotype rate that $82.4 \%$ (142) of cases would be genotyped at both markers of a marker-pair, on average across all between-gene pairs. The observed average was $85.3 \%$. Mean minor allele frequency in founders was 0.304 (range: 0.051-0.498). The mean expected frequency of minor allele-minor allele conjunctions over all marker pairs in cases was 0.0874 (range: $0.0023-0.2468$ ), and $\sim 64 \%$ of expected minor allele-minor allele pair frequencies were $>0.05$. The observed mean minor allele-minor allele frequency in cases was 0.0871 , with $71.0 \%$ being greater than 0.05 .

A total of 9269 SNP-SNP case-only interaction tests were performed within the 276 gene pairs. After exclusion of sparse tables, 9262 tests $(99.9 \%)$ remained. A QQ plot of the $\chi^{2}$ statistics showed some deflation of the statistics relative to the expected 1 d.f. $\chi^{2}$ distribution, with a 'stepped' appearance most likely due to nonindependence of tests (Supplementary Figure S2). Results are presented and sorted by the OR estimate or by $P$-value (Table 2 and Supplementary Table S3).

The largest OR observed was 7.27 between rs129882 in dopamine $\beta$-hydroxylase $(D B H)$ and $\mathrm{rs} 2283135$, a relatively rare variant in the SLC18A2 gene. Scaled to lie above 1, nine estimated interaction ORs exceeded 4.0, of which five included the rarest variant in the study: rs 916455 in DRD4, which had a minor allele frequency of 3\% in cases. For most of these, the bootstrap confidence interval calculation failed at either the upper or lower bound due to a sparse table. A total of 20 marker pairs showed an OR $>3.0$, including 12 pairs to which rs916455 contributed (Figure 1).
The smallest $P$-value observed was $5.4 \times 10^{-5}$ between rs11953285 and rs1611115 in the $A D R A 1 B$ and $D B H$ genes, respectively (Table 2). We note that no global $P$-value dipped below 0.97, between SLC18A2 and $D B H$, and all marker-pairs excluded from Table 2 showed a global $P$-value $=1$.

The conditional logistic regression approach yielded maximum pseudo- $\mathrm{R}^{2}$ values of 0.033 (partial model) to 0.045 (full model), indicating that a small fraction of the variance in affection status could be accounted for by the interaction terms. Correlation with the top case-only test results was strong (Spearman $\rho$ for $P$-values $=0.776$ ); over $80 \%$ of interaction ORs was in the same direction, ie, with the same allele (Supplementary Figure 5). Table 2 and Supplementary Table S3 show the results from the logistic regression.

\section{DISCUSSION}

From the study by Pierce and Ahsan, ${ }^{13}$ we estimate that a single pairwise case-only analysis with our sample size may have had good power to detect relatively large interaction relative risks $(>2)$, with interacting minor allele frequencies of $\sim 0.3$. Considering a range of minor allele frequencies from 0.05 to 0.5 , the least power would be obtained in the case where both interacting loci have risk alleles with frequency of 0.05 . In this case, $80 \%$ power would be obtained for an OR of 4.86 $(P<0.05)$. If one locus had risk allele frequency of 0.05 and the other locus $0.5,80 \%$ power is obtained at $\mathrm{OR}=2.24$. If both risk alleles had frequency 0.5 , an OR of 2.5 could be detected with $80 \%$ power at a significance level of $5.1 \times 10^{-6}$. Effects of these magnitudes are large by the standards seen to date in genetic association studies of ADHD; however, with the large number of tests between all markers in the candidate genes we examined, we did not expect to be able to achieve an acceptable gobal false-positive level nor in fact was this achieved. Therefore, we leave these findings as exploratory, to be used for prioritisation of future studies in independent samples. In a more detailed exploration of the interaction model (using, eg, a fourparameter model of additive $\times$ additive, additive $\times$ dominance,

Table 2 Top case-only interaction results $(P$-value $<0.005$ and $\mathrm{OR} \geq 2)$

\begin{tabular}{|c|c|c|c|c|c|c|c|c|c|c|}
\hline \multirow[b]{2}{*}{ Gene } & \multicolumn{2}{|l|}{ Locus 1} & \multicolumn{3}{|c|}{ Locus 2} & \multicolumn{2}{|c|}{ Case-only test } & \multicolumn{3}{|c|}{ Regression test } \\
\hline & Marker & Chr:pos & Gene & Marker & Chr:pos & Odds ratio $(95 \% \mathrm{Cl})$ & $\mathrm{P}$-value & Odds ratio & P-value & $\begin{array}{l}\text { Full model } \\
\text { pseudo- } R^{2 \mathrm{~b}}\end{array}$ \\
\hline$A D R A 1 B$ & rs11953285 & $5: 159324389$ & $D B H$ & rs1611115 & $9: 135490336$ & $4.56(2.10-20.02)$ & 0.000054 & 4.83 & 0.0004 & 0.018 \\
\hline SLC6A3 & Intron 8 VNTR & $5: 1464855$ & SNAP25 & rs362562 & 20:10 191186 & $2.41(1.46-4.23)$ & 0.000558 & 1.60 & 0.0871 & 0.008 \\
\hline ADRA1B & rs7737796 & $5: 159302007$ & SNAP25 & rs363006 & $20: 10228083$ & $2.43(1.50-4.37)$ & 0.000711 & 2.27 & 0.0102 & 0.016 \\
\hline ADRA1A & rs472865 & $8: 26754388$ & $D R D 4$ & exon 3 VNTR & 11:629989 & $3.64(1.61-14.41$ & 0.00100 & 3.80 & 0.0048 & 0.015 \\
\hline$A D R A 1 B$ & rs6884105 & $5: 159281021$ & SNAP25 & rs363006 & $20: 10228083$ & $2.18(1.38-3.85)$ & 0.00104 & 2.13 & 0.0215 & 0.008 \\
\hline ADRA1A & rs486354 & $8: 26766207$ & DRD4 & 120 bp duplication & $11: 625946$ & $2.46(1.42-5.21)$ & 0.00134 & 2.66 & 0.0111 & 0.012 \\
\hline$D B H$ & rs129882 & $9: 135513490$ & SLC18A2 & rs2283135c & 10:118989922 & $7.27(\mathrm{~N} / \mathrm{A})$ & 0.00178 & 9.45 & 0.0037 & 0.016 \\
\hline DRD4 & rs12720373 & $11: 626688$ & CES1 & rs3815589 & $16: 54384420$ & $2.47(1.38-5.36)$ & 0.00204 & 2.21 & 0.0222 & 0.015 \\
\hline DRD5 & D4S2928c & $4: 10612311$ & $D R D 4$ & exon 3 VNTR & $11: 629989$ & $5.84(N / A)$ & 0.00250 & 3.66 & 0.0664 & 0.007 \\
\hline CES1 & rs3815589 & $16: 54384420$ & ADRA1D & rs4815670 & $20: 4164864$ & $2.03(1.23-3.47)$ & 0.00314 & 2.03 & 0.0252 & 0.010 \\
\hline ADRA1A & rs472865 & $8: 26754388$ & $D B H$ & rs2797849 & 9:135491762 & $2.33(1.36-4.71)$ & 0.00328 & 2.28 & 0.0220 & 0.011 \\
\hline$A D R A 1 B$ & rs13162302 & $5: 159299853$ & $A D R A 1 D$ & rs6052456 & $20: 4173573$ & $2.67(1.41-6.64)$ & 0.00410 & 3.82 & 0.0010 & 0.021 \\
\hline DRD5 & D4S2928c & $4: 10612311$ & COMT & rs4680 & $22: 18331271$ & $2.60(1.38-5.27)$ & 0.00416 & 2.04 & 0.0904 & 0.004 \\
\hline HTR1B & rs1228814 & $6: 78230539$ & SLC6A4 & rs1872924 & $17: 25570472$ & $2.06(1.23-4.09)$ & 0.00456 & 3.24 & 0.0068 & 0.016 \\
\hline SLC18A2 & rs363279 & $10: 119026615$ & $S L C 6 A 2$ & rs9930182 & $16: 54298798$ & $3.38(1.59-13.27)$ & 0.00474 & 4.15 & 0.0033 & 0.017 \\
\hline
\end{tabular}

VNTR, variable number of tandem repeats polymorphism; chr:pos, chromosome:position (base pairs from pter; hg18).

Odds ratio were rescaled to lie above 1 , confidence intervals were derived from 1000 bootstrap samples, 'N/A' indicates that a ceiling or floor effect was observed in the bootstrap estimates owing to sparse contingency table; see Supplementary Materials for the full results.

Note: the $P$-values presented are unadjusted for multiple testing; none of them are significant at $P<0.05$ after correction for the number of tests.

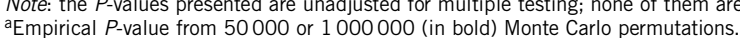

bMcFadden pseudo- $\mathrm{R}^{2}$ for the full model including main effects and interaction.

cMinor allele frequency $<0.1$ in parents. 


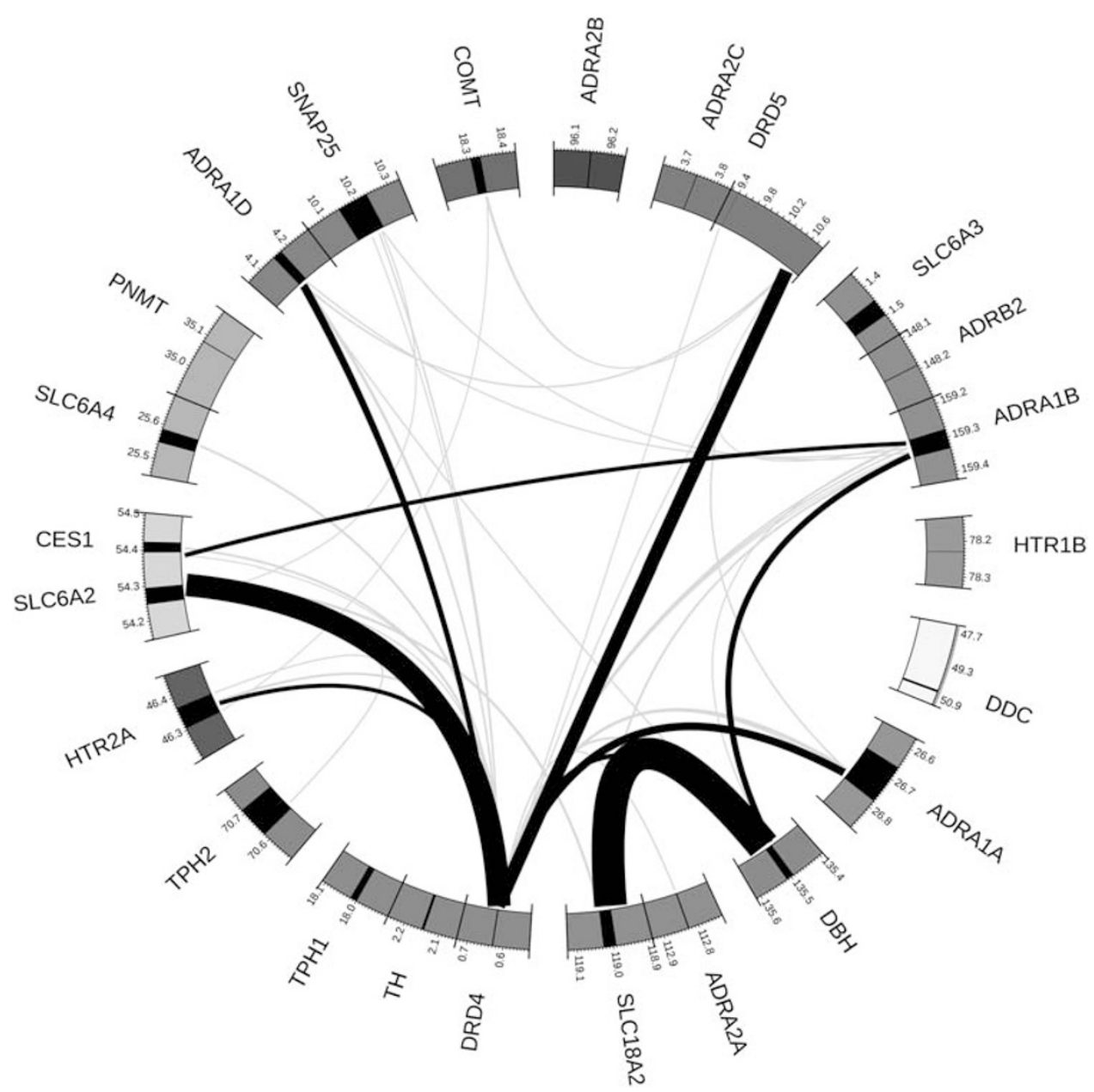

Figure 1 Visual representation of the interaction relative risk estimates for the top $0.5 \%$ of variant-variant interactions. Chromosomes are colour coded; gene spans in black. Note that the regions around the $D D C$ and $D R D 5$ genes are rescaled relative to other genes ( $1: 17$ and $1: 5$, respectively) owing to the distance to the furthest marker; interaction links are shaded by quantile: grey $=99.5-99.9$ th, black $\geq 99.9$ th; arc widths are proportional to the interaction OR.

dominance $\times$ additive, dominance $\times$ dominance), it was universally the case that unreliable parameter estimates were obtained for the top marker-pair interactions, and the model likelihood did not converge. This may simply reflect our modest sample size.

The presence of markers with a low MAF in the top interactions ranked by the ORs is expected because of unreliability of ORs derived from tables with small cell sizes as indicated by wide confidence intervals and as reflected in the $P$-values for these tests (see Supplementary Figure 4). When the OR is sensitive to small observed cell counts, the empirical $P$-value is of use to judge whether a particularly large estimate would be likely to occur by chance alone.

It is probable that several factors exist which preclude the independence of the individual pair-wise interaction tests performed with either the case-only design or the regression. These include linkage disequilibrium within genes because of ancestral co-segregation. We hope to have robustly excluded any effect owing to LD between syntenic markers by using a severe minimum distance between genes of $500 \mathrm{~kb}$. However, adjacent genes frequently share features at a functional level and in expression profile, ${ }^{23}$ and a large minimum distance may exclude some true functional interactions. Other potential confounders may also cause correlation between genes. These include, but are probably not limited to, higher order interaction effects on disease, on survival to the age of sampling, and membership of cryptic subpopulations. We have not tested the first of these owing to sample size limitations and to avoid a large increase in the number of tests and increased computational burden. Epistatic effects on survival to sampling cannot be excluded; however, no reports of marginal effects at these genes exist in the literature. Unfortunately, this sample was not genotyped at sufficient loci to allow an estimate of population stratification or to allow a genomic correction to be applied; however, the sample is ethnically homogeneous and therefore we do not expect any severe bias. This is supported by the strong correlation between the case-only and the family trio-based logistic regression results, which is not susceptible to the cryptic population structure in the sample.

As this study was intended to be hypothesis generating, no threshold defining statistical significance was enforced. Formal statistical evaluation of the evidence for interaction at the gene pair level is not trivial, as it would require consideration of both the number of markers within each gene and the extent of linkage disequilibrium, as well as higher order effects. Methods such as that of Chatterjee et $a l^{10}$ are of interest, but are not yet applicable to family-based or case-only designs. The LD patterns within each gene are relatively low (Supplementary Figure 1), and use of a gene-based permutation procedure that controls for LD will be important when a threshold for statistical significance is applied. 
The most studied gene-gene interaction in ADHD is for a joint effect of the DRD4 gene and the DA transporter gene (SLC6A3) VNTR polymorphisms, with some suggestive results. ${ }^{24-26}$ We were unable to demonstrate strong effects between DRD4 and SLC6A3. There is also a report in the literature of an interaction between the NA transporter (SLC6A2/NET1) and the COMT genes affecting quantitative traits derived from an ADHD self-report scale. ${ }^{27}$ Pairs of markers from these genes are amongst our top results ranked by $P$-value: $C O M T$ marker rs737866, when paired with three SLC6A2 markers, had ORs of 1.89 (95\% CI: $1.27-2.83 ; P=0.000866)$ with rs36017, 1.66 (1.14-2.41; $P=0.0085)$ with rs1345429, and $1.76(1.23-2.68 ; P=0.00128)$ with rs5569. These three SLC6A2 variants are relatively highly correlated with each other ( $r^{2}$ from 0.38 to 0.90 ; all $\mathrm{D}^{\prime}>0.97$ ), which may explain the correlation of ORs (see Supplementary materials, Figure $\mathrm{S} 1(\mathrm{O})$ ).

It has been hypothesised that functional disruption of serotoninrelated genes (SLC6A4, HTR1A, HTR1B) may act synergistically at the synapse to increase extracellular serotonin levels. ${ }^{28}$ We did observe modest interaction effects (Table 2) between two of these genes SLC6A4 and HTR1B (we did not examine HTR1A). Although this did not stand out as a finding in the case-only analysis, the logistic regression showed a large interaction OR estimate of 3.24 (Supplementary Table S3).

Two pairs of interactions with the smallest $P$-values stand out. $A D R A 1 B$ showed a minimum $P$-value of 0.00005 with $D B H$ $(\mathrm{OR}=4.56)$. DBH converts DA to NA, and in particular the SNP rs1611115 $(-1021 \mathrm{C} \rightarrow \mathrm{T})$ in the $\mathrm{DBH}$ gene promoter has been shown to have a strong effect on plasma enzyme activity. ${ }^{29}$ Here, the 'lowactivity' $\mathrm{C}$ allele (implying lack of NA and accumulation of DA) shows a synergistic effect with an $A D R A 1 B$ allele to increase risk. An interaction at or near this SNP could potentially explain some of the heterogeneous marginal association findings at this gene. ${ }^{1}$ There is a dearth of well-targeted studies on the ADRA1B gene product, and its central neural functions are unknown.

The second pair involves HTR2A with ADRA1A showing a minimum $P$-value of 0.0008 , with an $\mathrm{OR}=1.96$ (see Table 2, Supplementary Table S3). Linking these results, one publication has suggested that a HTR2A-mediated pathway may compensate for ADRA1B inactivity in a mouse hyperactivity model, ${ }^{30}$ raising the possibility that the products of these two genes may have some complementary function in a dopaminergic regulation that is also targeted by amphetamines (including methylphenidate).

Of course, a reasonable disease mechanism may be deduced from any subset of interactions that one selects, especially as these genes and polymorphisms were preselected for this very reason - this discussion on the potential functional relevance of these results is purely speculative, especially in the absence of known functional effects of the markers studied. However, these results have implicated a number of gene pairs with interesting effect sizes and suggestive of a functionally consistent set of interactions, which may converge on a common neurochemical phenotype, and which should be prioritised in future studies.

Although the detection of a statistical interaction may imply a biological (physico-chemical) interaction between the genes and/or gene products or via regulatory mechanisms, proof and further elucidation require extensive cell and molecular experiments. We also note that the statistical tests performed were exploratory, and we place greater emphasis on estimates of the effect sizes demonstrated here, which must, however, await replication in a larger, independent sample. Here, we were able to primarily investigate interaction in the form of a deviation from multiplicative effect on the risk ratio at pairs of markers in or near strong candidate genes. Interactions may lead to a marginal effect on disease risk at either or both of the loci; however, such marginal effects may appear considerably smaller than the true, unmeasured interaction, and therefore on a genome-wide scale power to detect such effects will be low, while controlling the family-wise error rate. For this reason, we feel that directed studies of interaction such as the present one may be fruitful when small but suggestive marginal effect sizes at the genes of interest have been demonstrated. This exploration of interactions between a selected set of candidate genes in our sample of ADHD cases has found some interesting results, justifying such further study.

\section{CONFLICT OF INTEREST}

The authors declare no conflict of interest.

\section{ACKNOWLEDGEMENTS}

We gratefully acknowledge the Health Research Board, Ireland for salaries and project funding (RS, ZH).

1 Gizer IR, Ficks C, Waldman ID: Candidate gene studies of ADHD: a meta-analytic review. Hum Genet 2009; 126: 51-90.

2 Li D, Sham PC, Owen MJ, He L: Meta-analysis shows significant association between dopamine system genes and attention deficit hyperactivity disorder (ADHD). Hum Mol Genet 2006; 15: 2276-2284.

3 Yang B, Chan RC, Jing J, Li T, Sham P, Chen RY: A meta-analysis of association studies between the 10-repeat allele of a VNTR polymorphism in the 3'-UTR of dopamine transporter gene and attention deficit hyperactivity disorder. Am J Med Genet B Neuropsychiatr Genet 2007; 144B: 541-550.

4 Neale BM, Medland SE, Ripke S et al: Meta-analysis of genome-wide association studies of attention-deficit/hyperactivity disorder. J Am Acad Child Adolesc Psychiatry 2010; 49: 884-897.

5 Elena SF, Lenski RE: Test of synergistic interactions among deleterious mutations in bacteria. Nature 1997; 390: 395-398.

6 Clark AG, Wang L: Epistasis in measured genotypes: Drosophila P-element insertions. Genetics 1997; 147: 157-163.

7 Komili S, Roth FP: Genetic interaction screens advance in reverse. Genes Dev 2007; 21: $137-142$.

8 Rio Frio T, Civic N, Ransijn A, Backmann JS, Rivolta C: Two trans-acting eQTLs modulate the penetrance of PRPF31 mutations. Hum Mol Genet 2008; 17: 3154-3165.

9 Cordell HJ: Detecting gene-gene interactions that underlie human diseases. Nat Rev Genet 2009; 10: 392-404.

10 Chatterjee N, Kalaylioglu Z, Moslehi R, Peters U, Wacholder S: Powerful multilocus tests of genetic association in the presence of gene-gene and gene-environment interactions. Am J Hum Genet 2006; 79: 1002-1016.

11 Autism Genome Project Consortium, Szatmari P, Paterson AD: Mapping autism risk loci using genetic linkage and chromosome rearrangements. Nat Genet 2007; 39: 319-328.

12 Neale BM, Laksy-Su J, Anney R et al: Genome-wide association scan of attention deficit hyperactivity disorder. Am J Med Genet B Neurospychiatr Genet 2008; 147B: 1337-1344

13 Pierce $\mathrm{BL}$, Ahsan $\mathrm{H}$ : Case-only genome-wide interaction study of disease risk, prognosis and treatment. Genet Epidemiol 2009; 34: 7-15.

14 Cordell HJ, Barratt BJ, Clayton DG: Case/pseudocontrol analysis in genetic association studies: a unified framework for detection of genotype and haplotype associations, gene-gene and gene-environment interactions, and parent-of-origin effects. Genet Epidemiol 2004; 26: 167-185.

15 Kirley A, Lowe N, Hawi Z et al: Association of the $480 \mathrm{bp}$ DAT1 allele with methylphenidate response in a sample of Irish children with ADHD. Am J Med Genet B Neuropsychiatr Genet 2003; 121B: 50-54.

16 Hawi Z, Lowe N, Kirley A et al: Linkage disequilibrium mapping at DAT1, DRD5 and DBH narrows the search for ADHD susceptibility alleles at these loci. Mol Psychiatry 2003; 8: 299-308.

17 Lowe N, Kirley A, Mullins C, Fitzgerald M, Gill M, Hawi Z: Multiple marker analysis at the promoter region of the DRD4 gene and ADHD: evidence of linkage and association with the SNP -616. Am J Med Genet B Neuropsychiatr Genet 2004; 131B: 33-37.

18 Hawi Z, Segurado R, Conroy J et al: Preferential transmission of paternal alleles at risk genes in attention-deficit/hyperactivity disorder. Am J Hum Genet 2005; 77: 958-965.

19 Kent L, Doerry U, Hardy E et al: Evidence that variation at the serotonin transporter gene influences susceptibility to attention deficit hyperactivity disorder (ADHD): analysis and pooled analysis. Mol Psychiatry 2002; 7: 908-912. 
20 Gatto NM, Campbell UB, Rundle AG, Ahsan H: Further development of the case-only design for assessing gene-environment interaction: evaluation of and adjustment for bias. Int J Epidemiol 2004; 33: 1014-1024.

21 O'Connell JR, Weeks DE: PedCheck: a program for the identification of genotype incompatibilities in linkage analysis. Am J Hum Genet 2998; 63: 259-266.

22 Yang Q, Khoury MJ, Sun F, Flanders WD: Case-only design to measure gene-gene interaction. Epidemiology 1999; 10: 167-170.

23 Hurst LD, Pál C, Lercher MJ: The evolutionary dynamics of eukaryotic gene order. Nat Rev Genet 2004; 5: 299-310.

24 Roman T, Schimtz M, Polanczyk G, Eizirik M, Rohde LA, Hutz MH: Attention-deficit hyperactivity disorder: a study of association with both the dopamine transporter gene and the dopamine D4 receptor gene. Am J Med Genet Neuropsychiatr Genet 2001; 195: 471-478.

25 Carrasco X, Rothhammer P, Moraga M et al: Genotypic interaction between DRD4 and DAT1 loci is a high risk factor for attention-deficit/hyperactivity disorder in Chilean families. Am J Med Genet B Neuropsychiatr Genet 2006; 141B: 51-54.
26 Henríquez $\mathrm{H}$, Henríquez M, Carasco X et al: Combination of DRD4 and DAT1 genotypes is an important risk factor for attention deficit disorder with hyperactivity families living in Santiago, Chile. Rev Méd Chile 2008; 136: 719-724.

27 Retz W, Rösler M, Kissling C et al: Norepinephrine transporter and catecholamine-Omethyltransferase gene variants and attention-deficit/hyperactivity disorder symptoms in adults. J Neural Transm 2008; 115: 323-329.

28 Stoltenberg SF: Epistasis among presynaptic serotonergic components. Behav Genet 2005; 35: 199-209.

29 Zabetian CP, Anderson GM, Buxbaum SG et al: A quantitative-trait analysis of human plasma-dopamine beta-hydroxylase activity: evidence for a major functional polymorphism at the DBH locus. Am J Hum Genet 2001; 68: 515-522.

30 Auclair A, Drouin C, Cotecchia S, Glowinski J, Tassin JP: 5-HT2A and alpha1badrenergic receptors entirely mediate dopamine release, locomotor response and behavioural sensitization to opiates and psychostimulants. Eur J Neurosci 2004; 20: 3073-3084.

Supplementary Information accompanies the paper on European Journal of Human Genetics website (http://www.nature.com/ejhg) 